\title{
Avaliação de sequências de treino para formação de classes de estímulos equivalentes com função comportamental especifica
}

\author{
Camila Maria Silveira Colodetti \\ Tereza Maria de Azevedo Pires Sério
}

\section{RESUMO}

O objetivo foi avaliar os resultados produzidos por sequências de treino para formação de classes de estímulos equivalentes, com função comportamental específica, que se diferem especialmente com relação ao primeiro treino realizado, a saber, discriminação simples ou condicional. Participaram da pesquisa 32 estudantes universitários. Metade deles foi submetida a uma sequência específica de treino que se iniciava com discriminação simples, e a outra metade foi submetida à outra sequência que se iniciava com discriminação condicional. Ambos os grupos passaram pelos dois procedimentos e pela reavaliação do desempenho nos testes das relações emergentes ou discriminação simples. Os resultados mostraram que iniciar o treino com a discriminação simples facilitou o desempenho dos participantes na discriminação condicional, assim como a discriminação condicional também facilitou o desempenho na discriminação simples. Por outro lado, os dados de reavaliação dos testes de equivalência indicaram que, para os participantes que não haviam atingido o critério na primeira aplicação desses testes, o treino em discriminação simples subsequente não gerou aumento nas porcentagens de acerto na reavaliação das relações emergentes.

Palavras-chave: equivalência de estímulos; equivalência funcional; sequência de treino.

\section{ABSTRACT}

\section{Evaluation of training sequences for formation of stimulus equivalence classes with specific behavioral function}

This research aimed to evaluate the results produced by training sequences for establish stimulus equivalence classes, with specific behavioral function, which differed especially with respect to the first training held, namely, simple or conditional discrimination. Participated in the study 32 college students. Half of these participants were exposed a specific training sequence that began with the simple discrimination, and the other half underwent another sequence that began with the conditional discrimination. Both groups were submitted to both procedures and to the reassessment of performance in emergent relations tests or simple discrimination tests. The results showed that starting with simple discrimination training facilitated participant's performance on conditional discrimination, as well as the conditional discrimination also facilitated performance on simple discrimination. On the other hand, the reassessment data of the equivalence tests indicated that, for the participants who did not reach the criterion in the first application of these tests, the subsequent training in simple discrimination did not increase the percentages of correctness in the reevaluation of the emergent relations.

Keywords: stimulus equivalence; functional equivalence; training sequence.

Pesquisas em equivalência de estímulos usualmente empregam o procedimento matchingto-sample para ensinar relações condicionais arbitrárias entre estímulos. Relações condicionais podem dar origem a classes de equivalência formadas por relações entre estímulos que não foram diretamente ensinadas. Para afirmar que os estímulos formam uma classe de estímulos equivalentes, a relação entre eles deve ser arbitrária de forma garantida e alguns critéri-

\section{Sobre as Autoras}

C.M.S.C.

orcid.org/0000-0001-5205-3890 Pontifícia Universidade Católica de São Paulo (PUCSP) - São

Paulo, SP

camilasilveira1@yahoo.com.br

T.M.A.P.S.

Pontifícia Universidade Católica de São Paulo (PUCSP) - São Paulo, SP

\section{Direitos Autorais}

Este é um artigo de acesso aberto e pode ser reproduzido livremente, distribuído,

transmitido ou modificado, por qualquer pessoa desde que usado sem fins comerciais. 0 trabalho é disponibilizado sob a licença Creative Commons CCBY-NC.

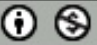




\section{MI* INTERACÃO EM LF PSICOLOGIA}

os formais precisam ser demonstrados: a relação entre os estímulos deve ser, ao mesmo tempo, reflexiva (um termo se relaciona com ele mesmo: por exemplo, se $A$ então $A$ ), simétrica (quando a ordem dos termos da relação é reversível: se $A$ então $B$; se $B$ então $A$ ) e transitiva (os termos iguais em dois pares, por exemplo, $A B$ e $B C$, determinam o terceiro par $A C)$. Para tanto, testes adicionais devem ser efetuados para verificar a existência de relações emergentes (de Rose \& Bortoloti, 2007; Sidman \& Tailby, 1982; Sidman, 1994).

Além de relações arbitrariamente definidas entre os estímulos, classes de estímulos também podem ser estabelecidas por meio de similaridade física entre os estímulos ou estabelecidas pela mediação de uma resposta comum. No primeiro caso, os estímulos dentro da classe são agrupados pelos seus atributos físicos e a classe delimitada pelas práticas de reforço da comunidade verbal (de Rose, 1993). No segundo caso, estímulos que evocam uma resposta comum constituiriam uma classe de estímulos funcional (de Rose 1993; Goldiamond, 1966; Sidman, 2000). Para de Rose (1993), além do compartilhamento de uma mesma resposta entre os estímulos que compõem a classe de estímulos, também seria necessária a demonstração de que variáveis aplicadas diretamente sobre um estímulo da classe têm efeito semelhante sobre os demais.

Alguns analistas do comportamento que pesquisam equivalência de estímulos têm se interessado pela interação entre procedimentos que propiciam o estabelecimento de classes de equivalência e de classes funcionais (de Rose, Mcllvane, Dube, Galpin, \& Stoddard, 1988; Montans \& Andery, 2009; Sidman, Wynne, Maguire, \& Barnes, 1989; Tyndall, Roche, \& James, 2004; Vaughan Jr., 1988). Pesquisas têm demonstrado ser possível a emergência de função de estímulos quando estímulos com função estabelecida por meio de discriminações simples são tornados membros de uma classe de equivalência (De Rose et al., 1988; Montans \& Andery, 2009; Tyndall et al., 2004). Outras têm demonstrado ser possível o estabelecimento de classes de estímulos equivalentes por meio de procedimentos que envolvem contingências de três termos (Sidman et al., 1989; Vaughan Jr., 1988).

Os autores de Rose et al. (1988) investigaram a emergência de discriminações simples em humanos, após uma sequência de procedimentos de discriminação simples e condicional. Cinco participantes receberam inicialmente o treino de discriminações simples A1/A2 e seis participantes receberam inicialmente o treino das discriminações condicionais $A B$. Nas tentativas $A 1 / A 2$, os estímulos A1 (S+) e A2 (S-) eram exibidos na tela de um computador e a posição do $\mathrm{S}+$ variava de forma não sistemática entre tentativas. Nas tentativas de discriminação condicional $A B$, os estímulos $A 1$ ou $A 2$ eram apresentados como estímulos modelo e os estímulos
B1 e B2 eram apresentados como estímulos comparação. Após o participante atingir o critério, começava a fase denominada pelos autores de "linha de base mista", na qual cada tipo de tentativa (discriminação simples ou condicional) era apresentada. Tentativas de sondas com o estímulo $B$, em que B1 e B2 apareciam sozinhos na tela do computador foram intercaladas entre as tentativas de linha de base mista. A seleção consistente do estímulo $\mathrm{B} 1$, nesse caso, indicaria se esses estímulos haviam adquirido funções similares aos estímulos A1 (S+) e A2 (S-).

Além desses procedimentos, foi também realizado um treino de reversão para quatro sujeitos, no qual a consequência para a escolha do estímulo $\mathrm{A} 2$ era correta e a do estímulo $A 1$, incorreta. As sondas do estímulo $B$ foram novamente introduzidas após esse treino. Foram realizados, ainda, os testes de simetria, nos quais $\mathrm{A} 1$ e $\mathrm{A} 2$ foram apresentados como estímulos comparação, e os estímulos B1 e B2 foram apresentados como estímulos modelo. Os resultados de todos os participantes que atingiram o critério de desempenho na linha de base mostraram que novos desempenhos de discriminação simples poderiam emergir sem treino explícito. Nas sondas dos estímulos $\mathrm{B}$, que não haviam participado do treino de discriminação simples, mas haviam sido relacionados condicionalmente aos estímulos $\mathrm{A}$, os sujeitos quase sempre selecionavam o estímulo que era condicionalmente relacionado ao $S+(A 1)$ nas tentativas A1/A2. Além disso, todos os quatro participantes que foram expostos à reversão das contingências $A 1 / A 2$ durante as sondas do estímulo $B$, selecionaram os estímulos condicionalmente relacionadas ao estímulo $\mathrm{A} 2$, que serviu como $\mathrm{S}+$ nesse treino. Segundo os autores os resultados sugeriram que B1 e B2 adquiriram funções similares aos estímulos $\mathrm{A} 1$ e $\mathrm{A} 2$, respectivamente, em virtude das relações de estímulos estabelecidas durante o treino $A B$.

Vaughan Jr. (1988) seguiu a direção inversa da proposta do estudo acima. 0 autor propôs que contingências de três termos poderiam estabelecer classes de estímulos equivalentes. Em seu estudo, seis pombos foram treinados a bicar slides contendo imagens de árvores. Os slides foram divididos em dois conjuntos, o positivo e o negativo. Na presença do conjunto positivo as bicadas eram reforçadas e na presença do negativo as bicadas não eram reforçadas. Após o critério ser atingido, as contingências eram revertidas, de modo que bicadas nos slides anteriormente negativos produziriam o reforçador, e bicadas nos slides anteriormente positivos não produziriam o reforçador. Os resultados mostraram que com o decorrer do treino ocorreu um aumento de respostas diante dos estímulos positivos e diminuição de respostas em relação aos estímulos negativos, e que após sucessivas reversões o responder alterava-se prontamente de acordo 
com a mudança nas contingências. Para o autor, esses resultados indicaram que os slides de cada um dos conjuntos passaram a controlar a mesma resposta dos pombos e que a similaridade funcional observada implicaria também no estabelecimento de relações de equivalência.

Sidman et al. (1989) discordaram da conclusão de Vaughan Jr. (1988) de que a similaridade funcional implicaria em relações de equivalência e propuseram um estudo para verificar se relações entre membros de uma classe funcional poderiam atingir os critérios que definem a equivalência de estímulos. Três humanos adultos que apresentavam patologias na fala participaram do estudo. Esses participantes foram inicialmente submetidos a uma série de discriminações simples simultâneas de duas escolhas e às reversões dessas discriminações. Após essa fase, os sujeitos foram submetidos a testes de discriminação condicional. No primeiro teste, verificou-se se os participantes poderiam emparelhar membros da mesma classe funcional. Depois, Sidman et al. (1989) ensinaram uma nova relação entre um dos estímulos membros das classes e dois novos estímulos e testaram as relações de simetria e transitividade.

Todos os participantes desse estudo, após aprenderem as discriminações simples, demonstraram a formação de classes de estímulos funcionais, uma vez que a reversão das contingências para um par de estímulos alterou as respostas a todos os outros pares. Além disso, os participantes demonstraram nos testes de discriminação condicional que os membros de cada classe funcional se relacionavam via equivalência. Após serem ensinados a relacionar novos estímulos a membros da classe já existente, dois dos três participantes foram capazes de emparelhar outros membros da classe aos novos estímulos. Inicialmente, Sidman et al. (1989) consideraram que o desempenho do único participante que falhou em demonstrar a inclusão de novos estímulos nas classes equivalentes era indicativo de que classes de estímulos funcionais e equivalentes eram estabelecidas por diferentes processos comportamentais, diferente do que foi afirmado por Vaughan Jr (1988). No entanto, em uma publicação posterior, Sidman (1994) revisou sua consideração e assumiu que o desempenho dos dois participantes que incluíram novos membros às classes equivalentes poderia indicar que as discriminações simples e as reversões dessas discriminações teriam estabelecido discriminações condicionais emergentes.

Montans e Andery (2009), também interessadas em investigar a interação entre ambos os processos, tiveram como objetivo testar a possibilidade de que um treino, envolvendo discriminações simples, sem reversão, pudesse produzir classes de estímulos equivalentes. As autoras tiveram como participantes seis crianças, submetidas primeiramente a um treino de discriminação simples entre três pares de estímulos (Conjuntos A, B e C). Após o treino de discriminação simples, foi realizado o treino de matching-to-sample entre D1 e A1, e D2 e A2. Testes foram conduzidos para verificar a emergência de relações condicionais e para verificar se os dois estímulos do conjunto D haviam adquirido as funções de S+ e S-. Os resultados mostraram que, três das seis crianças evidenciaram a formação de classes de estímulos equivalentes em todos os testes e o estabelecimento das funções de S+ e Sdos estímulos D. Segundo as autoras, a discriminação simples pareceu facilitar o estabelecimento da discriminação condicional devido ao número reduzido de blocos necessários para o treino de discriminação condicional.

Tyndall et al. (2004) consideram que um ponto de partida útil para a análise da relação entre equivalência de estímulos e equivalência funcional é o estabelecimento de funções de estímulos e o treino de relações de equivalência com esses mesmos estímulos. A facilitação, ou não, da formação de classes de equivalência com os estímulos que já apresentam função comportamental, de S+ ou S-, a priori poderia, então, auxiliar no entendimento da relação entre esses dois processos. Na pesquisa de Tyndall et al. (2004), participantes adultos foram primeiramente expostos a um procedimento de discriminação simples simultânea com sílabas sem sentido para que os estímulos $A 1, B 1, C 1, A 3, B 3$ e $C 3$ adquirissem a função de $S+$ e os estímulos $A 2, B 2, C 2, A 4, B 4$ e C4 adquirissem a função de $S$-. Na próxima fase, os participantes realizaram um procedimento de matching-to-sample para o estabelecimento de duas classes de estímulos equivalentes com três membros cada. Os participantes foram divididos em cinco condições que diferiam quanto aos estímulos que comporiam as classes de equivalência. Na condição $\mathrm{S}+$, o procedimento de treino empregou apenas os estímulos $\mathrm{S}+$. $\mathrm{Na}$ condição $\mathrm{S}+/ \mathrm{S}-$, as relações treinadas eram congruentes com as classes funcionais de $\mathrm{S}+\mathrm{e} \mathrm{S}$ - estabelecidas previamente (A1-B1-C1 e A4-B4-C4). Na condição mista S+/S-, treinou-se relações entre um estímulo $S+$ e dois estímulos $S-$, e entre um estímulo $\mathrm{S}$ - e dois $\mathrm{S}+$, de modo a formar classes que não se conformavam com as classes funcionais. $\mathrm{Na}$ condição S-, todos os estímulos que participaram do treino tinham função de S-. Por fim, na condição de não-função, os participantes não haviam realizado o treino de discriminação simples, mas realizaram o matching-to-sample para formação de duas classes de estímulos. Ao final do treino, testes de transitividade e simetria foram realizados.

Os resultados mostraram que na condição S+/S- os participantes formaram classes de equivalência mais rapidamente em relação às outras condições. Entretanto, para os autores, não se pode afirmar se os participantes, de fato, formaram classes de estímulos equivalentes na fase de teste, 
ou se simplesmente estabeleceu-se grupos funcionais de $\mathrm{S}+$ e S-. Em contraposição, foi na condição mista S+/S- que os participantes levaram mais tempo para formar classes de estímulos equivalentes. Também se observou que os participantes na condição não-função precisaram de mais blocos para formar classes de equivalência em relação aos participantes das condições S- e S+/S-. Segundo os autores, os resultados indicaram que a familiaridade com os estímulos de treino pode desempenhar algum papel na formação de classes de estímulos equivalentes.

Com base nos estudos relatados, é possível dizer que a relação entre função de estímulos e a emergência de relações de equivalência não é completamente compreendida. Estudos indicam que a equivalência funcional e a equivalência de estímulos interagem de modo a facilitar ou dificultar a formação de classes de estímulos (de Rose et al., 1986; Montans \& Andery, 2009; Sidman et al., 1989; Tyndall et al., 2004; Vaughan Jr., 1988). A presente pesquisa objetivou avaliar os resultados produzidos por sequências de treino para formação de classes de estímulos equivalentes, com função comportamental específica, que se diferiram especialmente com relação ao primeiro treino realizado, a saber, discriminação simples ou condicional. Incluíram-se, ainda, testes adicionais para verificar se, a depender do procedimento de discriminação (simples ou condicional) empregado primeiro, haveria modificação no desempenho dos participantes com relação às classes de estímulo estabelecidas.

\section{MÉTODO}

\section{PARTICIPANTES}

Participaram desse estudo 32 estudantes universitários de ambos os sexos. Escolheram-se, preferencialmente, estudantes de cursos diferentes da psicologia. 16 desses participantes foram submetidos a uma sequência de ensino de discriminações simples e condicionais e, outros 16 , a outra sequência. Os estudantes assinaram o termo de consentimento livre e esclarecido (O projeto foi aprovado pelo Comitê de Ética em Pesquisa da Pontifícia Universidade Católica de São Paulo: protocolo de pesquisa 261/2009).

\section{EQUIPAMENTO E MATERIAIS}

Foi utilizado um computador notebook e um software especialmente elaborado para esta pesquisa. O programa controlava a apresentação dos estímulos visuais e das consequências planejadas, bem como o registro das respostas. Foram utilizados nove estímulos visuais, disponíveis entre os símbolos do Microsoft Word 2007, distribuídos em três conjuntos de três estímulos cada.

\section{PROCEDIMENTO}

De modo geral, o procedimento envolveu treino de discri-

\begin{tabular}{|c|c|c|c|}
\hline Conjunto & & tímu & \\
\hline \multirow{2}{*}{ A } & A1 & $\mathrm{A} 2$ & A3 \\
\hline & $\Omega$ & $\%$ & $T$ \\
\hline \multirow{2}{*}{ B } & B1 & B2 & B3 \\
\hline & $\Xi$ & $\ddagger$ & $\beta$ \\
\hline \multirow{2}{*}{ C } & $\mathrm{C} 1$ & $\mathrm{C} 2$ & C3 \\
\hline & मே & $m$ & א \\
\hline
\end{tabular}

minação simples e condicional e testes referentes à formação de classes de equivalência e classes funcionais. Os participantes realizaram as tarefas no computador e deveriam clicar com o mouse sobre o estímulo correto. As consequências para as respostas foram: a apresentação da palavra "certo" quando a resposta era correta e da palavra "errado" quando a resposta era incorreta, acompanhada de um som característico de acertos ou erros. Além disso, havia escurecimento da tela por 1 segundo, quando a resposta era incorreta. 0 intervalo entre tentativas era de 0,5s.

Sequência 1. Para metade dos participantes, a sequência de treino para formação de classes de estímulos, denominada "Sequência 1", iniciou com o treino de discriminação simples seguido pelo treino de discriminação condicional e os testes propostos. As fases foram:

Fase 1: Discriminação simples simultânea. Essa fase teve como objetivo estabelecer as funções de S+ aos estímulos denominados A1, B1 e C1, e estabelecer as funções $S$ - aos estímulos A3, B3 e C3. Os estímulos A2, B2 e C2 não participaram do treino de discriminação simples. Pares de estímulos foram apresentados no centro da tela do computador. A posição na qual cada estímulo do par foi apresentado variava durante as tentativas. Primeiramente, eram apresentados os estímulos do conjunto A (A1 e A3) no centro da tela do computador, em blocos de 12 tentativas. 0 treino era encerrado assim que o critério de acerto de $90 \%$ fosse atingido. 0 treino com os pares de estímulos do conjunto $\mathrm{B}$ e do conjunto $\mathrm{C}$ ocorreu sucessivamente e de maneira idêntica à dos estímulos do conjunto $A$. Logo após, os pares de estímulos A1/A3, B1/B3 e C1/C3 eram apresentados em um mesmo bloco de 12 tentativas, sendo cada par apresentado quatro vezes. Quando o critério de $90 \%$ de acerto era alcançado, os pares de estímulos eram apresentados em blocos de 36 tentativas, contemplando todas as possíveis combinações entre os estímulos de todos os conjuntos, de forma que cada par fosse apresentado quatro vezes. Os participantes receberam feed- 


\section{H. INTERACÃO EM * PSICOLOGIA}

back por acertos e erros em todas as tentativas.

Fase 2: Discriminação condicional. Essa fase consistiu de quatro etapas cujo objetivo foi treinar relações condicionais arbitrárias entre estímulos. 0 procedimento utilizado foi o matching-to-sample com zero de atraso. Um clique no estímulo modelo com o mouse resultava no desaparecimento desse estímulo e no aparecimento dos três estímulos comparação. 0 critério para encerramento de cada etapa do treino foi $100 \%$ de acerto em um bloco. A apresentação dos estímulos seguiu os seguintes critérios: a mesma relação condicional foi apresentada, no máximo, por duas vezes consecutivas; todas as posições de escolha na tela acolheram o mesmo número de estímulos com reforçamento programado; a ocorrência de reforçamento em uma dada posição não poderia ocorrer mais de duas vezes consecutivas.

Etapa 1. Procedimento de emparelhamento com o modelo AB. Esta etapa foi composta por blocos de 36 tentativas, nos quais o estímulo modelo apresentado era um dos três estímulos $A$, e os estímulos comparação eram os estímulos $B$. Era fornecido feedback para todas as tentativas.

Etapa 2. Procedimento de emparelhamento com o modelo BC. 0 procedimento foi o mesmo da etapa 1. Contudo, os estímulos modelo eram os estímulos do conjunto $\mathrm{B}$ e os estímulos apresentados como alternativa de escolha eram os estímulos do conjunto $\mathrm{C}$. Era fornecido feedback para todas as tentativas.

Etapa 3. Blocos de revisão. Nessa etapa, os participantes foram submetidos a blocos de 24 tentativas mixadas, das relações $A B$ e $B C$ treinadas anteriormente. Era fornecido feedback para todas as tentativas.

Etapa 4. Linha de base em reforçamento intermitente. 0 objetivo dessa etapa foi familiarizar o participante com as condições de extinção semelhantes à etapa de teste das relações emergentes. Foram apresentados blocos de 24 tentativas das relações condicionais treinadas nas etapas anteriores. Em apenas $50 \%$ das tentativas a escolha dos estímulos pelo participante produzia feedback.

Fase 3: Testes de simetria, transitividade e transitividade simétrica. 0 objetivo dessa fase foi verificar a emergência das relações de equivalência. Foram testadas a emergência das relações simétricas $\mathrm{BA}$ e $\mathrm{CB}$, a emergência da relação transitiva $A C$, e a emergência da relação de equivalência $C A$, para verificar se os estímulos $A, B$ e $C$ formaram uma classe de estímulos equivalentes. 0 conjunto de testes foi composto por 24 tentativas nas quais as quatro relações testadas eram apresentadas seis vezes. Não havia consequências para acertos e erros. 0 participante prosseguia para a próxima fase, caso tivesse atingido $80 \%$ de acerto.

Fase 4: Reavaliação da discriminação simples, sem refor- çamento. Essa fase teve como objetivo verificar se o procedimento de discriminação condicional não interferiu na discriminação simples inicialmente estabelecida. Para tanto, eram novamente apresentados os pares de estímulos do procedimento de discriminação simples simultânea. Esses pares eram apresentados em 27 tentativas, totalizando três apresentações de cada um dos nove pares possíveis.

Sequência 2. A outra metade dos participantes foi submetida a uma sequência de treino diferente, denominada "Sequência 2". O procedimento era idêntico para cada fase e etapa, com exceção da Fase 4, na qual novos testes de equivalência eram realizados ao invés da retomada da discriminação simples. A ordem da sequência de treino foi alterada da seguinte forma: Fase 1 - Discriminação condicional; Fase 2 - Testes de equivalência; Fase 3 - Discriminação simples simultânea; Fase 4 - Reavaliação dos testes de equivalência. A Fase 4 foi idêntica à Fase 3 da Sequência 1 e à Fase 2 da Sequência 2.

\section{RESULTADOS}

Participaram deste estudo 32 estudantes universitários. 16 deles foram submetidos à sequência 1 de treino, iniciando pela discriminação simples e, os outros 16 , à sequência 2 , que se iniciava com a discriminação condicional. 25 participantes apresentaram as relações emergentes esperadas no teste de equivalência já na primeira sessão de teste e outros sete participantes precisaram passar pelo retreino das relações. Dos sete participantes que precisaram de retreino, dois haviam sido submetidos à Sequência 1 e cinco à Sequência 2.

0 critério estabelecido para o teste de equivalência foi um total de acertos de pelo menos $80 \%$. Na Figura 1 é apresentada a quantidade de participantes por porcentagem de acertos nos últimos testes de equivalência, após retreino das relações para aqueles participantes que não atingiram o critério nas primeiras sessões de teste

15 participantes que foram submetidos à Sequência 1 de treino apresentaram mais de $86 \%$ de acerto nesses testes. Apenas um participante, que realizou essa Sequência de treino, apresentou $79 \%$ (faixa de 76 a $85 \%$ de acertos na Figura 1). A porcentagem de acertos no teste de equivalência em cada uma das Sequências foi próxima. Os dados permitem concluir, no que diz respeito ao teste de relações emergentes, que não há uma sequência de treino que favoreça esse desempenho quando ambas são comparadas. Na Tabela 2 é apresentada, para a Sequência 1 e 2, a quantidade de participantes por número de blocos e de erros ocorridos até que o critério na discriminação simples fosse atingido. 


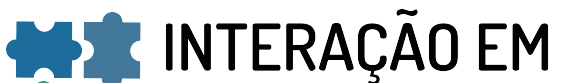 ETS PSICOLOGIA}

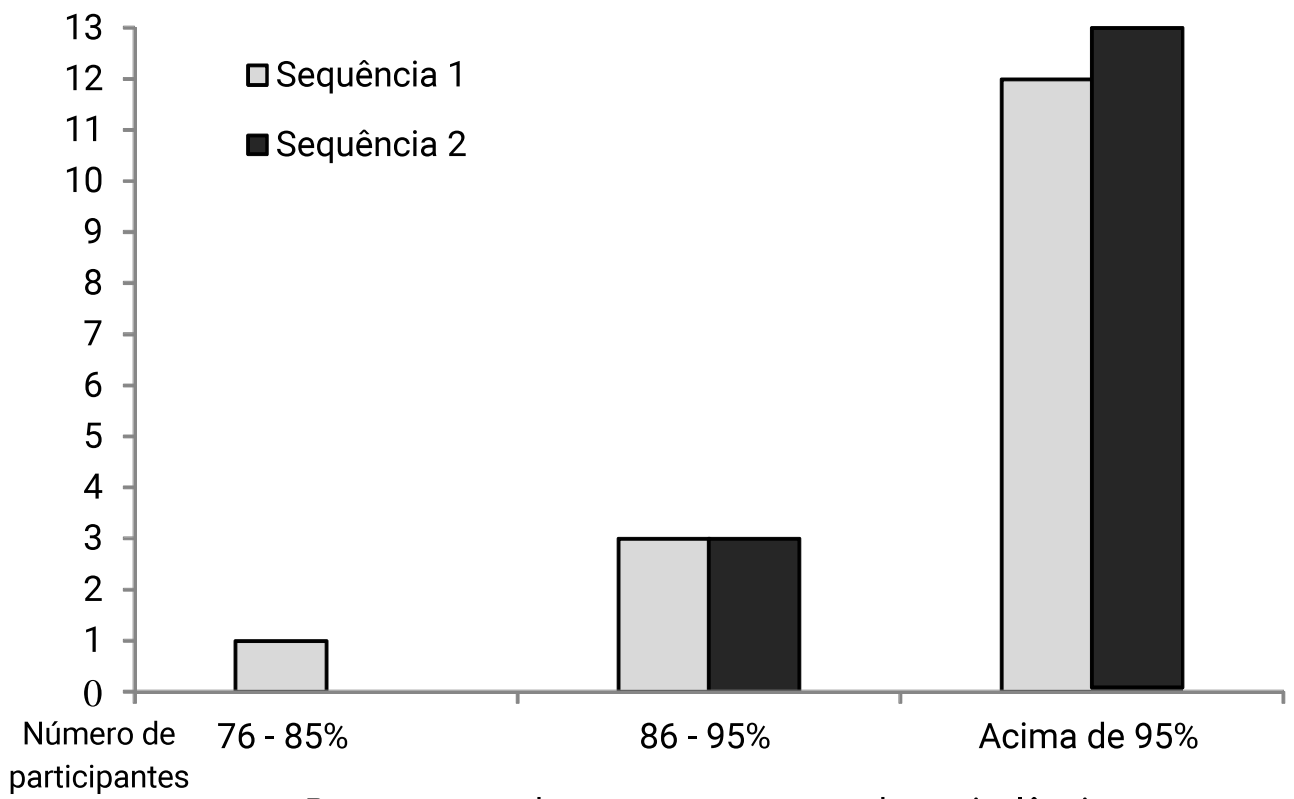

Porcentagem de acertos nos testes de equivalência

Figura 1. Quantidade de participantes por porcentagem de acertos nos testes de equivalência.

De modo geral, a maioria dos participantes expostos previamente ao procedimento de discriminação condicional (Sequência 2) parece atingir antes e com menos erros o critério para considerar estabelecida a discriminação simples. Dos 16 participantes submetidos a essa sequência, 12 precisaram da quantidade mínima de blocos (5) para atingir esse critério. Dos 16 participantes que iniciaram o experimento com a discriminação simples (Sequência 1), apenas oito precisaram dessa mesma quantidade de blocos. Além disso, a faixa de erro para os participantes que iniciaram o treino com a discriminação condicional (Sequência 2) variou de 0 a 5 , sendo que a maioria dos participantes (6) apresentou apenas um erro na discriminação simples. Em contrapartida, a faixa

Tabela 2. Quantidade de participantes das sequências 1 e 2 por quantidade de blocos e de erros na discriminação simples.

\begin{tabular}{cccccccccc}
\hline & & \multicolumn{1}{c}{$N^{\circ}$} & \multicolumn{1}{c}{ Número de erros } \\
\cline { 3 - 9 } De treino & blocos & 0 & 1 & 2 & 3 & 4 & 5 & 6 & 7 \\
\hline 1 (discriminação & 5 & - & 2 & 4 & 1 & 1 & - & - & - \\
simples $\rightarrow$ & 6 & - & - & 1 & 2 & 1 & 2 & - & - \\
condicional) & 7 & - & - & - & - & - & 1 & - & 1 \\
\hline 2 (discriminação & 5 & 3 & 6 & 1 & 2 & - & - & - & - \\
condicional $\rightarrow$ & 6 & - & - & 1 & 2 & - & 1 & - & - \\
simples) & 7 & - & - & - & - & - & - & - & - \\
\hline
\end{tabular}

de erros dos participantes da Sequência 1, que começaram o treino com o procedimento de discriminação simples, variou de 1 a 7 erros.

Como a maioria dos participantes que passaram pelo treino de discriminação condicional antes da discriminação simples precisou de um menor número de blocos e apresentou menor quantidade de erros na discriminação simples, é possível que a discriminação condicional possa ter facilitado o desempenho neste treino.

A seguir estão apresentadas as figuras com relação ao número de blocos necessários para atingir o critério de $100 \%$ de acerto nas relações $A B$ (painel da esquerda) e $B C$ (painel da direita), por número de participantes.

Com base na Figura 2 (painel da esquerda), é possível observar que, com relação à discriminação condicional $A B$, de modo geral, os participantes da sequência que se iniciou com a discriminação simples (Sequência 1) precisaram de menos blocos que os participantes da sequência que se iniciou com a discriminação condicional (Sequência 2). Quatorze participantes da Sequência 1 precisaram de até três blocos para atingir $100 \%$ de acerto, sendo que desses 14,10 precisaram de dois blocos e um participante precisou somente de um bloco para atingir o critério, o que indica que ele não cometeu erros. Com relação à Sequência 2, 10 participantes precisaram de até três blocos para atingir o critério, sendo que desses dez, sete precisaram de dois blocos e três precisaram de três blocos. 

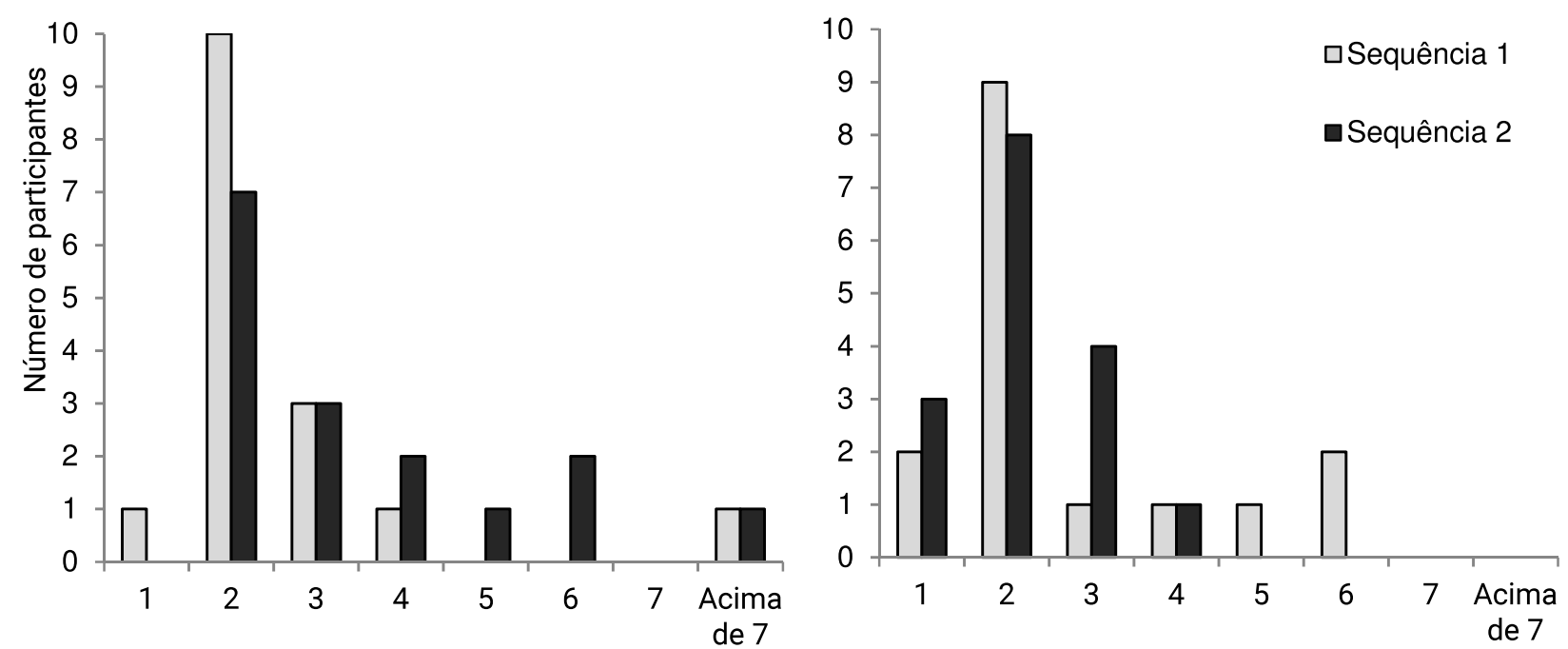

Figura 2. Quantidade de participantes, em cada uma das sequências, por quantidade de blocos $A B$ e $B C$ necessários para atingir o critério de $100 \%$ de acerto na discriminação condicional. $O$ painel da esquerda mostra os resultados para a relação $A B$ e o painel da direita os resultados para a relação $B C$.

Com relação ao número de blocos $\mathrm{BC}$ necessário para atingir o critério (Figura 2, painel da direita), os participantes da sequência que se iniciou com a discriminação condicional (Sequência 2) precisaram de um número menor de blocos em comparação ao número de blocos necessários para o treino da relação $A B .15$ participantes da Sequência 2, precisaram de no máximo três blocos, e apenas um precisou de quatro blocos. Além disso, diferentemente do que foi observado para o treino $A B$, há três participantes da Sequência 2 , que não cometeram nenhum erro no primeiro bloco $\mathrm{BC}$, uma vez que precisaram realizar somente um bloco para atingir $100 \%$ de acerto. Com relação aos participantes da Sequência 1 , o número de blocos necessários para para o treino das relações $A B$ e $B C$ foi bastante próxima. Para o treino da relação $\mathrm{BC}$, doze participantes precisaram de até três blocos, e somente três precisaram de cinco ou seis blocos para atingir o critério.

Os participantes da Sequência 1, que já haviam sido expostos à discriminação simples apresentaram melhor desempenho no treino da relação $A B$, em relação aos participantes da Sequência 2. Além disso, houve participantes de ambos os grupos que apresentaram melhor desempenho no treino da segunda discriminação condicional (BC), se comparado ao desempenho no treino da primeira relação condicional AB. Três participantes da Sequência 2 e dois participantes da Sequência 1 não apresentaram erros no treino da relação $\mathrm{BC}$, precisando de apenas um bloco para atingir o critério.

A seguir serão apresentados resultados com relação à re- avaliação da discriminação simples para os participantes da Sequência 1, e com relação à reavaliação do teste de equivalência para os participantes da Sequência 2, nas Tabelas 3 e 4, respectivamente.

A Tabela 3 apresenta os resultados da reavaliação da discriminação simples, reinserida após o teste de equivalência. Todos os participantes para prosseguir no procedimento haviam atingido $90 \%$ de acertos no treino. A tabela apresenta também o número de blocos que os participantes precisaram para atingir o critério no treino.

14 dos 16 participantes apresentaram $75 \%$ de acertos ou mais, e desses 14 , dez acertaram mais de $90 \%$ nas tentativas. Há dois participantes que diminuíram notavelmente a por-

Tabela 3. Porcentagem de acertos na reavaliação da discriminação simples.

\begin{tabular}{|c|c|c|c|c|c|}
\hline \multirow{2}{*}{$\begin{array}{c}\% \text { de } \\
\text { acertos } \\
\text { para } \\
\text { critério no } \\
\text { treino }\end{array}$} & \multirow{2}{*}{$\begin{array}{l}N^{\circ} \text { de } \\
\text { blocos } \\
\text { para o } \\
\text { critério }\end{array}$} & \multirow{2}{*}{$\begin{array}{c}\text { No }^{\circ} \\
\text { part. }\end{array}$} & \multicolumn{3}{|c|}{$\begin{array}{c}\text { \% de acertos na } \\
\text { reavaliação da } \\
\text { discriminação simples }\end{array}$} \\
\hline & & & $\begin{array}{l}\text { Abaixo } \\
\text { de } \\
75 \%\end{array}$ & $\begin{array}{c}75- \\
89 \%\end{array}$ & $\begin{array}{l}\text { Acima } \\
\text { de } \\
90 \%\end{array}$ \\
\hline \multirow[t]{3}{*}{$90 \%$} & 5 & 8 & 1 & 3 & 4 \\
\hline & 6 & 6 & 1 & 1 & 4 \\
\hline & 7 & 2 & & & 2 \\
\hline
\end{tabular}




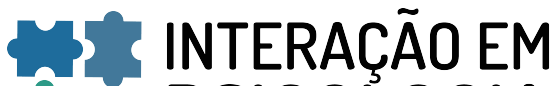 ET PSICOLOGIA}

centagem de acertos e apresentaram abaixo de $75 \%$ de acertos logo após terem passado pelo treino de discriminações simples e pelos testes de relações emergentes. Também vale destacar que os dois participantes que precisaram do número maior de blocos (7) mantiveram a porcentagem de acertos acima de $90 \%$ na reavaliação do desempenho.

Na Tabela 4 é possível verificar o desempenho dos participantes da Sequência 2 na reavaliação do teste de equivalência realizado após o procedimento de discriminação simples. A maioria dos participantes (14 de 16) atingiu mais de $75 \%$ de acertos. Desses 14 participantes, 11 apresentaram mais de $90 \%$ de acertos no primeiro teste, antes do treino de discriminação simples, e mantiveram o desempenho no segundo teste, após o treino da discriminação simples. Dois dos 16 participantes apresentaram porcentagem de acertos abaixo de $75 \%$ no primeiro teste de equivalência, e mantiveram o desempenho na reavaliação dos testes de equivalência, a despeito de terem passado pelo treino de discriminação simples antes do segundo teste de equivalência. Para esses participantes, o treino de discriminação simples não facilitou na emergência de relações condicionais. Dos três participantes que haviam apresentado de $75-89 \%$ de acertos, um manteve o desempenho no teste de relações emergentes após treino de discriminação simples e o outro reduziu a porcentagem de acertos.

De modo geral, aqueles participantes que apresentavam desempenho próximo de $100 \%$ no primeiro teste de equivalência mantiveram esse desempenho posteriormente, mas aqueles que haviam apresentado próximo de $75 \%$ de acertos

Tabela 4. Porcentagem de acertos na reavaliação dos testes de equivalência

\begin{tabular}{|c|c|c|c|c|}
\hline \multirow{2}{*}{$\begin{array}{l}\% \text { de acertos no } \\
\text { primeiro teste de } \\
\text { equivalência }\end{array}$} & \multirow{2}{*}{$\begin{array}{c}\mathbf{N}^{\circ} \\
\text { part. }\end{array}$} & \multicolumn{3}{|c|}{$\begin{array}{c}\% \text { de acertos na } \\
\text { reavaliação nos testes de } \\
\text { equivalência }\end{array}$} \\
\hline & & $\begin{array}{l}\text { Abaixo } \\
\text { de } 75 \%\end{array}$ & $\begin{array}{l}75- \\
89 \%\end{array}$ & $\begin{array}{l}\text { Acima } \\
\text { de } 90 \%\end{array}$ \\
\hline Abaixo de $75 \%$ & 2 & 2 & & \\
\hline $75-89 \%$ & 3 & 2 & 1 & \\
\hline Acima de $90 \%$ & 11 & & & 11 \\
\hline
\end{tabular}

diminuíram a porcentagem de acertos na reavaliação, indicando que, para eles, o treino de discriminação simples não facilitou no estabelecimento das relações emergentes.

\section{DISCUSSÃO}

Os resultados mostraram que o primeiro treino de relações entre estímulos facilitou o segundo treino. A maioria dos participantes que passou pelo treino de discriminação condicional antes do treino de discriminação simples apresentou menor quantidade de erros e menor número de blocos na discriminação simples, e vice-versa, como é possível verificar nos dados apresentados na Tabela 2 e na Figura 2. Estudos anteriores demonstraram que o treino discriminativo poderia facilitar o estabelecimento de relações condicionais entre estímulos (Montans \& Andery, 2009; Tyndall et al., 2004). Tyndall et al. (2004) demonstraram que o treino de discriminações condicionais em conformidade com classes funcionais estabelecidas anteriormente estabeleceu mais rapidamente classes de equivalência.

0 presente estudo mostrou que a direção de treino inversa, com o treino de discriminações condicionais precedendo o treino de discriminação simples, também facilitou este último treino, conforme os resultados apresentados na Tabela 2. Estudos têm demonstrado que depois de estabelecidas classes funcionais, o treino de um novo estímulo via discriminação condicional tem sido suficiente para que este adquira a função dos estímulos aos quais foi emparelhado (Montans \& Andery, 2009; Sidman et al., 1989). Se por meio da relação de condicionalidade é possível verificar transferência de função entre estímulos, não é surpreendente que o procedimento de discriminação condicional tenha facilitado o desempenho da discriminação simples. Mesmo porque as classes de estímulos treinadas, assim como na condição S+/S- do estudo de Tyndall et al. (2004), eram concordantes com a função de estímulo estabelecida para cada membro da cada classe, de modo que $\mathrm{A} 1, \mathrm{~B} 1$ e $\mathrm{C} 1$ formavam a classe de S+ e A3, B3 e C3 formavam a classe de S-. Além disso, não é possível descartar que a própria familiaridade com os estímulos já estabelecida no primeiro treino pode ter sido uma condição de facilitação do desempenho no segundo treino (Tyndall et al., 2004).

Em 1994, Sidman considerou que uma contingência de três termos pode levar à emergência de relações de equivalência. Em seu estudo de 1989 com outros autores, dois dos três participantes demonstraram em testes subsequentes de discriminação condicional que os membros de cada classe funcional se relacionavam via equivalência. Com base nos resultados obtidos no presente estudo, no entanto, pode-se afirmar que classes de equivalência foram formadas, mas não é possível afirmar se classes funcionais foram estabelecidas, haja vista que a transferência de função entre os membros não foi testada. No entanto, considerando o estudo de 


\section{H. INTERACÃO EM LF PSICOLOGIA}

Sidman et al. (1989) questiona-se se é possível que os participantes da Sequência 1, que se inicia com o procedimento de discriminação simples, tenham simplesmente estabelecido classes funcionais.

Os resultados obtidos também podem ser discutidos à luz do conceito de learning set, relativo à aprendizagem de uma nova discriminação ser melhor após uma história de exposição a outros treinos discriminativos (Catania, 1999/2006; Harlow, 1949). O efeito de um treino de discriminação sobre uma nova aprendizagem não foi apenas observado na facilitação do treino de discriminação simples pelo treino de discriminação condicional e vice-versa, mas também na melhora do desempenho no segundo bloco de discriminação condicional, especialmente para os participantes cujo primeiro treino foi em discriminação condicional. Pelo menos oito participantes da sequência que se iniciou com a discriminação condicional apresentaram melhor desempenho no treino da segunda relação (BC), conforme se observa na Figura 2.

Em termos da rota de ensino empregada, autores sugerem que o ensino de discriminações deva iniciar por discriminações simples (Dube, 1996). De fato, iniciar por discriminação simples reduz o número de erros em discriminações condicionais, mas a recíproca também é verdadeira. Talvez o teste das sequências de treino em outra população de participantes que não estudantes universitários possa levar a diferentes resultados, tendo em vista que Dube (1996) discute sequências de ensino de discriminações para crianças que apresentam atraso no desenvolvimento.

Com relação à manutenção do desempenho na discriminação simples, os dados mostraram que dois participantes diminuíram a porcentagem de acertos. Um deles precisou do número mínimo de blocos no treino para atingir o critério. Dos dois participantes que precisaram de dois blocos a mais para atingir o critério no treino, o desempenho se manteve (acima de 90\% de acertos) na reavaliação da discriminação simples, indicando que um treino com mais repetições pode auxiliar no estabelecimento das relações. Além disso, para esses participantes que diminuíram a porcentagem de acertos na reavaliação da discriminação simples não se descarta, também, a possibilidade do controle pelo estímulo S-. Embora não tenham sido mostrados desempenhos individuais, vale a pena citar que um desses participantes pareceu ter revertido as classes quase por completo, selecionando em mais de $90 \%$ das tentativas os estímulos não relacionados ao reforçamento. Outro aspecto importante a ser considerado na redução do desempenho desses participantes é que imediatamente antes da reavaliação da discriminação simples, eles haviam sido submetidos aos testes das relações emergentes em extinção. É também possível que o fato de dois testes seguidos terem sido realizados, sem reforçamento, tenha perturbado o desempenho desses participantes.

Na reavaliação do teste de equivalência, dois participantes haviam apresentado pouco menos de $75 \%$ no primeiro teste de equivalência e mantiveram o desempenho na reavaliação, a despeito de terem passado pelo treino de discriminação simples imediatamente antes. Outros dois participantes tinham apresentado de $75 \%$ a $89 \%$ no primeiro teste de relações emergentes e reduziram esse desempenho após o treino de discriminação simples. De acordo com Sidman (1987), embora o critério de $75 \%$ seja significativamente acima do acaso, é possível que o participante tenha desempenhado consistentemente para algumas relações e não para outras. Segundo o autor, até mesmo com um critério de $90 \%$ o participante poderia acertar $100 \%$ das tentativas das relações $A B$ e $80 \%$ das relações BC. Embora 25 dos 32 participantes do estudo tenham apresentado acima de $95 \%$ de acerto nos testes de equivalência, alguns prosseguiram adiante com o critério próximo de $75 \%$, o que explica a redução ou manutenção do desempenho nos testes de reavaliação das relações emergentes para os participantes citados. No entanto é interessante notar que os participantes seguiram adiante no procedimento a despeito de estarem abaixo do critério a fim de se verificar se o treino de discriminações simples poderia auxiliar na emergência das relações condicionais. Mas o que se observou foi o contrário: os participantes com critério acima de $90 \%$ no primeiro teste mantiveram o desempenho, mas dos cinco participantes que apresentaram menor porcentagem de acertos, não houve aumento na porcentagem de acertos e, em alguns casos, a porcentagem foi reduzida.

Os resultados do presente estudo referentes à facilitação do desempenho durante o treino de discriminação condicional após o treino de discriminação simples, assim como um desempenho com menos erros e menor quantidade de blocos na discriminação simples após o treino de discriminação condicional favorece a interpretação de que os processos envolvidos em ambos os casos poderiam estar intimamente relacionados. Por outro lado, os dados que indicam que a discriminação simples não propiciou o aumento nas porcentagens de acerto nos testes de relações emergentes para os participantes que tinham inicialmente falhado em demonstrar essas relações, pode indicar que ambos são processos comportamentais distintos. De qualquer maneira, essa é uma área de investigação que merece atenção, dado que a relação entre equivalência de estímulos e equivalência funcional não é completamente compreendida. 


\section{W'INTERACÃO EM ET. PSICOLOGIA}

\section{CONTRIBUIÇÃO DE CADA AUTOR}

T.M.A.P.S. foi responsável pela supervisão da pesquisa em todas as suas fases, sendo orientadora de C.M.S.C. durante a sua formação como mestre em Análise do Comportamento. C.M.S.C. foi responsável por todas as etapas da pesquisa, sob orientação de T.M.A.P.S.

\section{DECLARAÇÃO DE CONFLITOS DE INTERESSES}

Os autores declaram que não há conflitos de interesse no presente artigo.

\section{REFERÊNCIAS}

Catania, A. C. (2006). Aprendizagem: Comportamento, linguagem e cognição. (D. G. de Souza, Trad.). Porto Alegre: Artmed. (Trabalho original publicado em 1999).

de Rose, J. C. (1993). Análise comportamental da cognição. Psicologia: Teoria e Pesquisa, 9(2), 283-303.

de Rose, J. C., \& Bortoloti, R. (2007). A equivalência de estímulos como modelo do significado. Acta Comportamentalia, 15, 83-102.

de Rose, J. C., Mcllvane, W. J., Dube, W. V., Galpin, V. C., \& Stoddard, L. T. (1988).

Emergent simple discrimination established by indirect relation to differential consequences. Journal of the Experimental Analysis of Behavior, 50(1), 1-20.

Dube, W. V. (1996). Teaching discrimination skills to person with mental retardation. In C. Goyos; M. A. Almeida \& D. de Souza (Orgs.), Temas em Educação Especial (pp. 73-96). São Paulo: EDUFSCar.

Goldiamond, I. (1966). Perception, language and conceptualization rules. In B. Kleinmuntz (Ed.), Problem Solving: Research, Method, and Theory. Nova York: Wiley
Camila Maria Silveira Colodetti e Tereza Maria de Azevedo Pires Sério

Harlow, H. F. (1949). The formation of learning sets. Psychological Review, 56, 51-65.

Montans, M. P. S., \& Andery, M. A. P. A. (2009). A emergência de relações condicionais entre estímulos como resultado de treino de série de discriminações simples simultâneas. Estudos de Psicologia, 26(2), 133-146.

Sidman, M. (1987). Two choices are enough. Behavior Analysis, 22(1), 11-18.

Sidman, M. (1994). Equivalence relations and behavior: A research story. Boston: Authors Cooperative.

Sidman, M. (2000). Equivalence Relations and the Reinforcement Contingency. Journal of the Experimental Analysis of Behavior, 74, 127-146.

Sidman, M. \& Tailby, W. (1982). Conditional discrimination vs. matching- to- sample: An expansion of the testing paradigm. Journal of the Experimental Analysis of Behavior, 37, 5-22.

Sidman, M., Wynne, C. K., Maguire, R. W., \& Barnes, T. (1989). Functional classes and equivalence relations. Journal of the Experimental Analysis of Behavior, 52(3), 261-274.

Tyndall, I. T., Roche, B., \& James, J. E. (2004). The relations between stimulus function and equivalence class formation. Journal of the Experimental Analysis of Behavior, 81 (3), 257-266.

Vaughan Jr., W. (1988). Formation of equivalence sets in pigeons. Journal of Experimental Psychology: Animal Behavior Processes, 14(1), 36-42. 